\title{
Heartbeat: Recreational substance use and risk of premature atherosclerotic cardiovascular disease
}

doi:10.1136/heartjnl-2021-319322

The potential association of recreational substance use and premature atherosclerotic cardiovascular disease (ASCVD) is controversial. In this issue of Heart, Mahtta and colleagues performed a cross-sectional analysis of 135703 patients with premature ASCVD (first event at age $<55$ years for men and $<65$ years for women) compared with over 1 million patients with non-premature ASCVD using the 2014-2015 US nationwide Veterans Affairs Healthcare database and Veterans with permaTure AtheroscLerosis (VITAL) registry. ${ }^{1}$ Patients with premature ASCVD, compared with non-premature ASCVD, had higher use of tobacco $(62.9 \%$ vs $40.6 \%)$, alcohol (31.8\% vs $14.8 \%)$, cocaine $(12.9 \%$ vs $2.5 \%)$, amphetamine $(2.9 \%$ vs $0.5 \%)$ and cannabis $(12.5 \%$ vs $2.7 \%)(\mathrm{p}<0.01$ for all comparisons). The odds of premature ASCVD were higher in those using recreational substances and there was a graded response relationship between the number of substances used and a higher likelihood of premature ASCVD. The magnitude of risk was greater in women compared with men (figure 1).

As Scott, Murnane and Orr point out in the accompanying editorial, "Retrospective studies are limited by the available data. While this study supports the association between substance use disorder and early-onset ASCVD, the effect of substance use frequency, dose and duration cannot be reliably ascertained in this patient sample." Even so, these findings suggest that public education is needed on the potential longterm risk of ASCVD with recreational substance use, as well as counselling of individuals patients. We might also consider earlier screening for ASCVD in young adult patents with a history of a substance use disorder.

Another interesting study in this issue of Heart examined the effect of socioeconomic disparities on intervention and survival after out-of-hospital

Correspondence to Professor Catherine M Otto, Division of Cardiology, University of Washington, Seattle, Washington, USA; cmotto@uw.edu

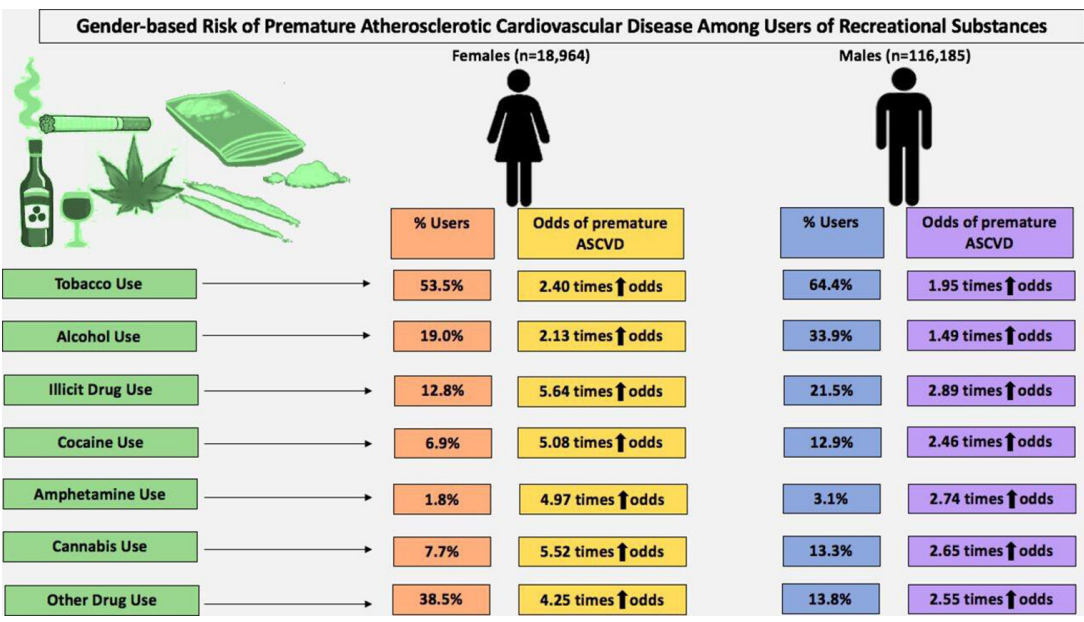

Figure 1 Gender-Based risk of premature atherosclerotic cardiovascular disease (ASCVD) among users of recreational substances.
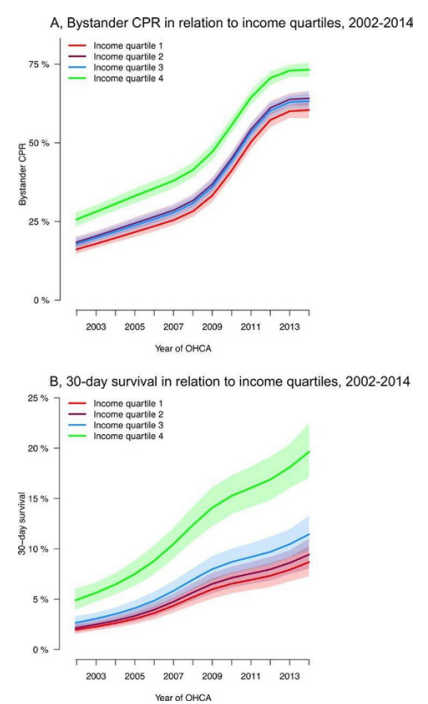

Figure 2 Temporal trend in bystander CPR and 30-day survival in relation to income quartiles, 2001-2014. logistic regression analyses for temporal trends in bystander CPR (A) and 30-day survival (B) following OHCA in relation to income quartiles, standardised after sex and age (in 10 year intervals). $p<0.001$ for all groups for trends over time. no significant interaction between income group and year was observed. CPR, cardiopulmonary resuscitation; OHCA, out-of-hospital cardiac arrest. cardiac arrest (OHCA). ${ }^{3}$ In the 21480 patients in the Danish Cardiac Arrest Registry over age 30 years from 2001 to 2014, those in the highest income quartile were younger, with higher education and less comorbidities relative to the lowest income quartile. In addition, the highest income quartile had a 26\% higher survival for public witnessed OHCA compared with the lowest income patients. Although rates of bystander cardiopulmonary resuscitation (CPR) were lower in lower income patients, the predicted effect of this difference in bystander CPR on survival was less than $1 \%$ and the survival difference related to socioeconomic status not explained by other identifiable patient or cardiac-arrest related factors. Improvements in both bystander CPR and survival were seen over time for all income levels, but with persistent socioeconomic differences (figure 2).

To explain these findings, Noordman, Blaauw and Maass ${ }^{4}$ argue that it is the quality of CPR, not just whether it was performed, that is critical for survival. They propose that "it is of major importance to improve the quality of bystander intervention, especially among people with a relatively low 

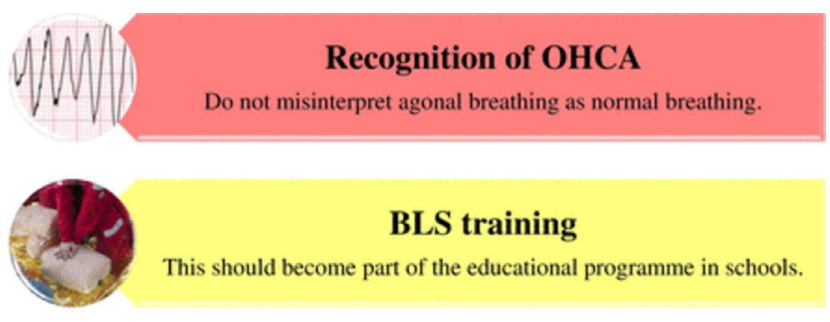

BLS training

This should become part of the educational programme in schools.
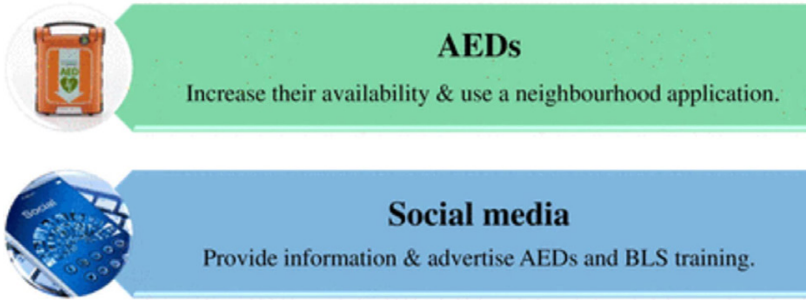

Figure 3 Four steps for the improvement of the quality of bystander intervention. if successfully implemented, these steps have the potential to increase survival following sudden cardiac death in people of all socioeconomic positions. AED, automated external defibrillator; BLS, basic life support; OHCA, out-of-hospital cardiac arrest.

level of education and socioeconomic status" starting with the steps shown in figure 3. We applaud their stated goal: "let us use education to create a world wherein sudden cardiac death survival does not depend on one's socioeconomic position."

Cardiologists increasingly care for patients who have received potentially cardiotoxic mediations for treatment of cancer. Tyrosine kinase inhibitors (TKIs) improve survival in patients with chronic myeloid leukaemia (CML) but the risk of major adverse cardiovascular events (MACE) with these agents is uncertain. In a population based retrospective cohort of over 4000 patients with CML, compared with over 40 thousand matched controls, the incidence of MACE was similar in those with or without CML from 2001 onwards. ${ }^{5}$ However, there was a persistent higher risk of cerebrovascular events (8.6 vs 5.6 per 1000 personyears; $1.35,95 \%$ CI 1.00 to 1.83 ) and peripheral arterial events (6.9 vs 3.0 per 1000 person-years; $1.6695 \% \mathrm{CI}$, 1.15 to 2.39 ) in patients with $\mathrm{CML}$ compared with patients without CML, even after 2001 (figure 4). There were no differences between TKIs.

As this study illustrates, we need both more cardio-oncologists in the community and a greater awareness of the interplay between cancer therapies and cardiovascular disease by all cardiologists "when cancer therapy becomes part of a chronic condition" as so eloquently discussed by Zhang. ${ }^{6}$ "By targeting general cardiologists as part of its educational purview, the cardio-oncology community has the opportunity to greatly amplify its educational reach and thereby optimise the long-term cardiovascular care of patients with cancer on a larger scale."

The Education in Heart article in this issue $^{7}$ reviews the evolution of the "Heart Team"concept, summarises the advantaged and challenges of the current heart team model, and propose future directions "to meet the demands of a changing landscape of complex coronary and valvular heart disease (figure 5)."

Our series of review articles on Cardiogenetics continues in this issue of Heart with a detailed and practical approach to genetic testing in the diagnosis and management of patients with aortic disease by Thakker and Braverman. ${ }^{8}$ Now that genetic testing for inherited aortopathies is widely available it is important to recognise, as the authors conclude, that "Genetic testing can establish a specific diagnosis in about $25 \%$ of cases of familial
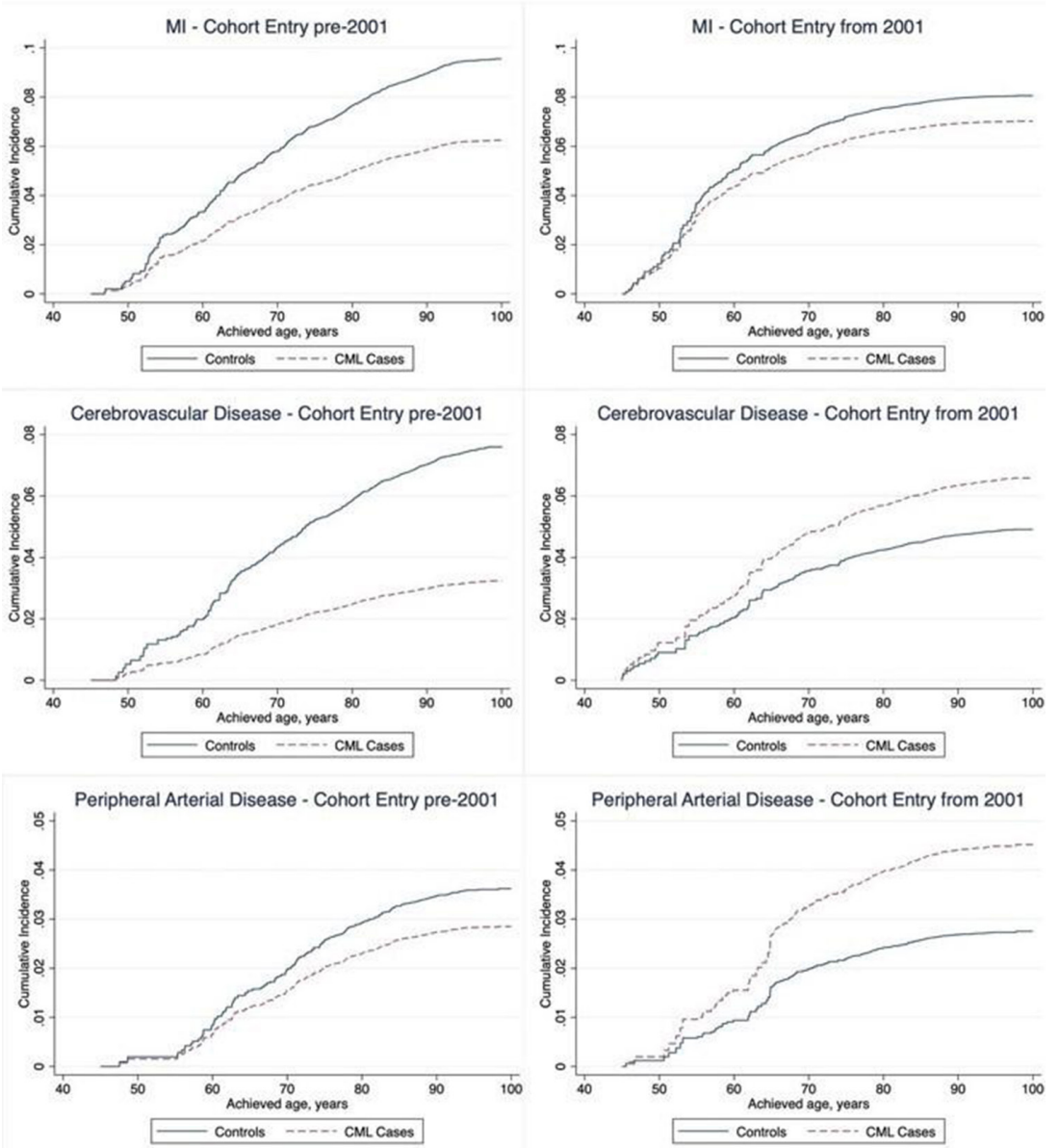

Figure 4 Cumulative incidence of myocardial infarction (MI), cerebrovascular events and peripheral arterial disease events stratified by cohort entry before 2001 vs from 2001 and by chronic myeloid leukaemia (CML) cases vs controls, adjusted for age; sex; history of $\mathrm{MI}$, cerebrovascular disease, peripheral arterial disease, heart failure, diabetes, hypertension; chronic obstructive pulmonary disease and rural vs urban location. 


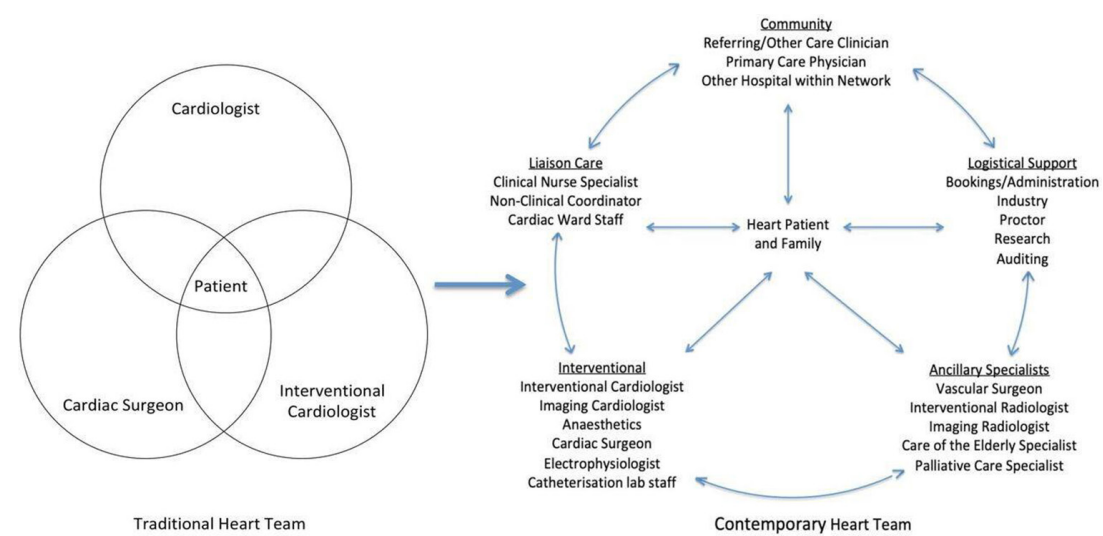

Figure 5 The traditional TAVI heart team and a proposed composition of the 'perfect' modern valve heart team. TAVI, transcatheter aortic valve implantation.

aortic disease and a genetic diagnosis can guide medical and surgical management. Regardless of whether a specific gene is discovered, screening of family members is important and will identify others with aortic disease in 20\% of cases of TAA or dissection."

Funding The authors have not declared a specific grant for this research from any funding agency in the public, commercial or not-for-profit sectors.

Competing interests None declared.
Patient and public involvement Patients and/or the public were not involved in the design, or conduct, or reporting, or dissemination plans of this research.

Patient consent for publication Not required.

Provenance and peer review Commissioned; internally peer reviewed.

(c) Author(s) (or their employer(s)) 2021. No commercial re-use. See rights and permissions. Published by BMJ.

\section{Check for updates}

To cite Otto CM. Heart 2021;107:599-601.
Heart 2021;107:599-601.

doi:10.1136/heartjnl-2021-319322

ORCID iD

Catherine M Otto http://orcid.org/0000-0002-05279392

\section{REFERENCES}

1 Mahtta D, Ramsey D, Krittanawong C, Rifai MA, et al. Recreational substance use among patients with premature atherosclerotic cardiovascular disease. Heart 2021;107:650-6.

2 Scott ML, Murnane KS, Orr AW. Young at heart? drugs of abuse cause early onset cardiovascular disease in the young. Heart 2021;107:604-6.

3 Møller S, Wissenberg M, Starkopf L. Socioeconomic disparities in pre-hospital factors and survival after out-of-hospital cardiac arrest. Heart 2021;107:627-34

4 Noordman A, Blaauw Y, Maass AH. Successful resuscitation of sudden cardiac death: education matters. Heart 2021;107:602-3.

5 Leong D, Aghel N, Hillis C, et al. Tyrosine kinase inhibitors in chronic myeloid leukaemia and emergent cardiovascular disease. Heart 2021:107:667-73.

6 Zhang KW. Out of the woods, but still in the weeds: when cancer therapy becomes part of a chronic condition. Heart 2021;107:607-8.

7 Yeoh J, MacCarthy P. Is it time to refresh the heart team? new paradigms for shared decision making. Heart 2021;107:674-81.

8 Thakker PD, Braverman AC. Cardiogenetics: genetic testing in the diagnosis and management of patients with aortic disease. Heart 2021;107:619-26. 